\title{
Selecting Environments for Distribution of Services
}

\section{Kambiz Farahmand ${ }^{1}$, Satpal Singh Wadhwa ${ }^{1 *}$, Mahmoud Mostafa ${ }^{1}$ and Vahid H Khiabani ${ }^{2}$}

${ }^{1}$ North Dakota State University, USA

${ }^{2}$ Middle Tennessee State University, USA

\begin{abstract}
Aim: The aim of this study is to provide a mechanism for decision makers in health services to compare the possible solutions for distribution of specialty services in a given area and to further compare the specialty services for dominant solutions.

Background: There is an increasing recognition that modelling tools can assist in the process of health care decision making. It is imperative that the managers should have some tools at their disposal for efficiently determining what needs to be done in order to reach the best decision they could reach with the information and knowledge that they have.

Methods: In this paper, we propose a questionnaire for expanding healthcare specialty services in a given geographic area. Excel serves as a user interface as well as a powerful computing program. This tool evaluates one or more specialty service to be distributed using three proposed solutions/methods for expanding the specialty service.

Results and discussion: The proposed decision making tool will be an easy assessment tool that could be used by VA (Veteran Affairs) managers and decision makers to decide if the specialty service(s) is an ideal candidate for distribution. The decision making tool will be in the form of a questionnaire and the answers to questions will be scored and if the overall score is above the threshold, then the specialty service will be considered a good candidate for expansion or distribution. In summary, we believe the tool adds value to the scarce existing literature on decisionmaking tools for service distribution in healthcare.
\end{abstract}

Keywords: Healthcare decision making; Decision making tool; Specialty service distribution; Evidence based design tool; Healthcare data analytics

\section{Introduction}

A specialist is a doctor who, as the name implies, specializes in a particular type of medicine, like dermatology, podiatry and orthopedics etc. Through Veteran Affairs (VA) specialty care services, you have access to expert knowledge to optimize treatment in unique or complicated courses of care. Their specialty care providers focus on particular areas of care in which they have extensive training and education.

In order to decide if a specialty service (SS), offered as part of a healthcare program for a targeted group of patients such as U.S. Department of Veterans Administration (VA) offering specialty services for veterans, may be a good candidate for distribution into the rural geographic location several factors must be carefully analyzed and evaluated. For example, the distribution should be based on VA criteria's and the veterans / patient criteria's for distribution as well as maximizing access for veterans as stated by the Veterans Choice Program. The proposed decision making tool will be an easy assessment tool that could be used by VA managers and decision makers to decide if the specialty service(s) is an ideal candidate for distribution. The decision making tool will be in the form of a questionnaire which will allow the decision makers to determine and evaluate basic requirements for distribution of services. The answers to the questionnaire will be scored and if the overall score is above the threshold, then the specialty service will be considered a good candidate for expansion or distribution.

There are four main solutions to the distribution problem; 1) distributing to an existing facility; 2) using a third party provider; 3 ) building a new facility; or 4) a combination of some of them. Each solution constitutes a category of questions that are designed to assess the feasibility of the solution for each specialty service. Each specialty service has a score for each question under the categories designated for each solution. Hence, the goal is to designate the best qualitative solution for each specialty service, and then to compare the specialty services and their respective solutions quantitatively based on cost, distance travelled by patient, and the potential for long term sustainability. The best solutions may further be compared on the basis of various characteristics for the purpose of acquiring a well characterized view of the whole system and its interaction with the other characteristics of the system.

The Veterans Choice Program for example attempts to maximize access for veterans who has had a difficult time getting an appointment with their designated provider due to geographic or other reasons. These veterans will now have a choice to receive non-VA health care rather than waiting for a VA appointment or traveling to a VA facility.

Beginning November 5, 2014, the new Choice Program began to cover non-VA care for eligible Veterans enrolled in VA healthcare. Veterans are eligible if the following conditions apply

- Veterans have been told by their local VA medical facility that they will need to wait more than 30 days from their preferred date or the date medically determined by their physician

- Their current residence is more than 40 miles from the closest VA health care facility

- They need to travel by plane or boat to the VA medical facility closest to their home

*Corresponding author: Satpal Singh Wadhwa, North Dakota State University IME, NDSU Dept. 2485, PO Box 6050 Fargo, ND 58102, United States, Tel: +17013532136; E-mail: satpalsingh.wadhwa@ndsu.edu

Received February 02, 2016; Accepted February 17, 2016; Published February 25, 2016

Citation: Farahmand K, Wadhwa SS, Mostafa M, Khiabani VH (2016) Selecting Environments for Distribution of Services. Health Care: Current Reviews 4: 159 doi: $10.4172 / 2375-4273.1000159$

Copyright: (C) 2016 Farahmand K, et al. This is an open-access article distributed under the terms of the Creative Commons Attribution License, which permits unrestricted use, distribution, and reproduction in any medium, provided the original author and source are credited. 
- They face an unusual or excessive burden in traveling to a VA medical facility based on the presence of a body of water (including moving water and still water) or a geologic formation that cannot be crossed by road

\section{Literature Review}

Anthony D. Bristol in his thesis talks about hiring a third party health care provider to perform a task previously and traditionally done in-house [1]. The search for financial performance, quality improvement and fiscal accountability are the possible reasons for outsourcing these healthcare services. But the incomparable nature of healthcare services with other commodities raises several administrative and policy issues for the management to contemplate outsourcing.

His thesis provides an overview of how the outsourcing phenomenon is being practiced as a strategic management tool. This thesis outlines a methodology that devises an outsourcing scale that organizations can utilize to ensure the most strategic option for outsourcing.

Zadeh et al. proposed a framework to develop the evidence based tool in the stages of planning, design and construction and operation stages of healthcare facilities [2]. This framework lays a common platform of analytical language for the stakeholders, by pairing EBD with value-driven decision making. They used engineering economy tools to evaluate the return on investments. His research concluded that the Evidence-based and value-based design frameworks can address the issues of life-cycle costs and savings of EBD to stakeholders and thereby results in more informed decision making and optimization of healthcare infrastructure.

Zhu et al. in their paper are talking about intelligent healthcare systems [3]. They developed a human-centric user model to bridge the gap between the patients and the doctor. The model consists of ten major concepts including physical, character, belief, capacity, experience, physical-context, character-context, belief-context, capacity context, and experience-context. These inherited characteristics can help to tell the doctor what kind of healthcare the patient wants to acquire, namely patient's desire. By utilizing such an approach, the intelligent healthcare system is able to acquire patient's desire within three steps: 1) Learning the semantic user model from extrinsic information resources of patient. 2) Learning the semantic user model by reasoning intrinsic concepts, instances and relationships. 3) Reasoning patient's desire based on patient's semantic user model.

Beer et al. interprets the health environment with the help on an agents based environment that allows managers, administers, clinicians and others to judge the effects of their decision both on patients and other providers [4]. Professionals working in groups take on a specific role in the health economy, with some specific objectives (expressed in terms of income and expenditure, waiting lists, and patient satisfaction) and resources. They pursue these objectives through interaction with other roles. These are mapped onto agents in the system, which can either act as Proxies for professionals in the exercise. The agreed results of any decisions, in terms of the system and individual parameters and then fed back to the whole system. When all the decisions are available, the agent simulation runs, and results are available for the next session. Users register with the system, they then interact with their agent which provides both the key indicators of the current status of the simulation. The professionals are expected to review the current situation at the start of each turn, and then decide the changes they wish to make in order to balance the income expenditure and the quality of care.

Jun GT et al. in their paper has a summarized description of a limited number of distinct modelling methods and evaluate how healthcare workers perceive them. Eight distinct modelling methods were identified and characterized by what the modelling elements in each explicitly represents [5]. Flowcharts, which had been most extensively used by the participants, were most favored in terms of their usability and utility. However, some alternative methods, although having been used by a much smaller number of participants, were considered to be helpful, specifically in understanding certain aspects of complex processes, e.g. communication diagrams for understanding interactions etc. They found that it is important to make the various process modelling methods more easily accessible to health care by providing clear guidelines or computer-based tool support for health care-specific process modelling.

Jun et al. [6] aimed to provide a mechanism for decision makers in health services planning and management to compare a broad range of modelling and simulation methods so that they can better select and use them or better commission relevant modelling and simulation work. They developed comparison and selection tool in which 28 different methods were identified and characterized by their relevance to different application areas, project life cycle stages, types of output and levels of insight, and four input resources required (time, money, knowledge and data). This tool makes a contribution in two major ways. The first is to fill a research practice gap in evidence-based health care management by providing a practical support for the method comparison and selection. Not only can the tool help health care professionals commission more appropriate modelling work, but may also assist health care modelling consultants and researchers to expand their modelling repertoire in order to meet the diverse needs of their health care clients.

\section{Limitations of the Previous Studies}

All the previous studies in one way or the other contributing to the improvement of healthcare. Anthony D Bristol devised an outsourcing scale to choose the third party health care provider to perform a task previously and traditionally done in-house. But this is not the only option to improve quality of care or to improve the financial performance [1]. There are other options like distributing the services to the existing facility and building a new facility, all of which needs to be compared and then the best amongst them.

Zadeh et al. proposed a framework to develop the tool for the optimization of healthcare infrastructure [2]. His tool is more cost and profit centric, not incorporating the quality and continuity of care, availability and reach of the healthcare facility to the patients.

Beer et al. interpret the health environment with the help on an agents based simulation that allows health care workers to take decisions [4]. The results of the decisions are fed into the simulation programmer, which runs and then provides the result for the next cycle. This is indeed is a good method to see the effect of the decision making, but firstly the decisions are not backed up by information and evidence and secondly this tool is not handy as all the healthcare workers are not competent to take decisions and skillful to operate the simulation program.

In this paper we devised a tool which can be used by the easily healthcare managers, as it only requires MS office excel, which is widely available and easy to use. In this tool we compared four solutions 1) distributing to an existing facility; 2) using a third party provider; 3 ) building a new facility; or 4) a combination of some of them, to find the best candidate for specialty service. This tool is novel of its kind 
as we not only had a balance between the cost and quality of care but also included the measures of availability and proximity of a healthcare facility for a patient in a geographic area.

\section{Methodology}

The Specialty Services (SS) were compared to see which is more suitable for the four options of either constructing a new facility, distributing services to other facilities, using third party providers, or a combination of these solutions. The comparison is done using a series of multiple choice questions. The user specifies a Minimum Requirement (MR) for each question. The MR is the minimum accepted answer, and any answer below this MR would receive a negative score. If the SS score differently on a given question than MR, the difference between the answers is given to the SS as an "advantage score". Hence, the worst answer for a given category will always set to be choice \# 1 and the best answer will always be choice \# 5 ; insuring that the better (positive) score will reflect an answer that is better than the MR while the worse (negative) score will reflect an answer that is worse than the MR.

Consider an example (see Figure 1), if the answer for SS was 7,000 and the MR specified by the user was 500 , then the advantage score would be equal to 2 for that SS (Specialty Service). Next, the user would rate the importance of the parameter that the question is concerned with.

The advantage score then will be multiplied by the "Importance Rating". Hence, if I rate the importance of "Maximum demand" in determining my choice for distributing SS to be 5, then the "Question Score" is advantage score multiplied by Question Importance Rating = $2 \times 5=10$ for SS1(Specialty Service 1). After determining a question score for each question and the SS that the question score belongs to, we should have an idea about which SS is suitable for distribution using which solution. At this point, the scoring procedure is done and

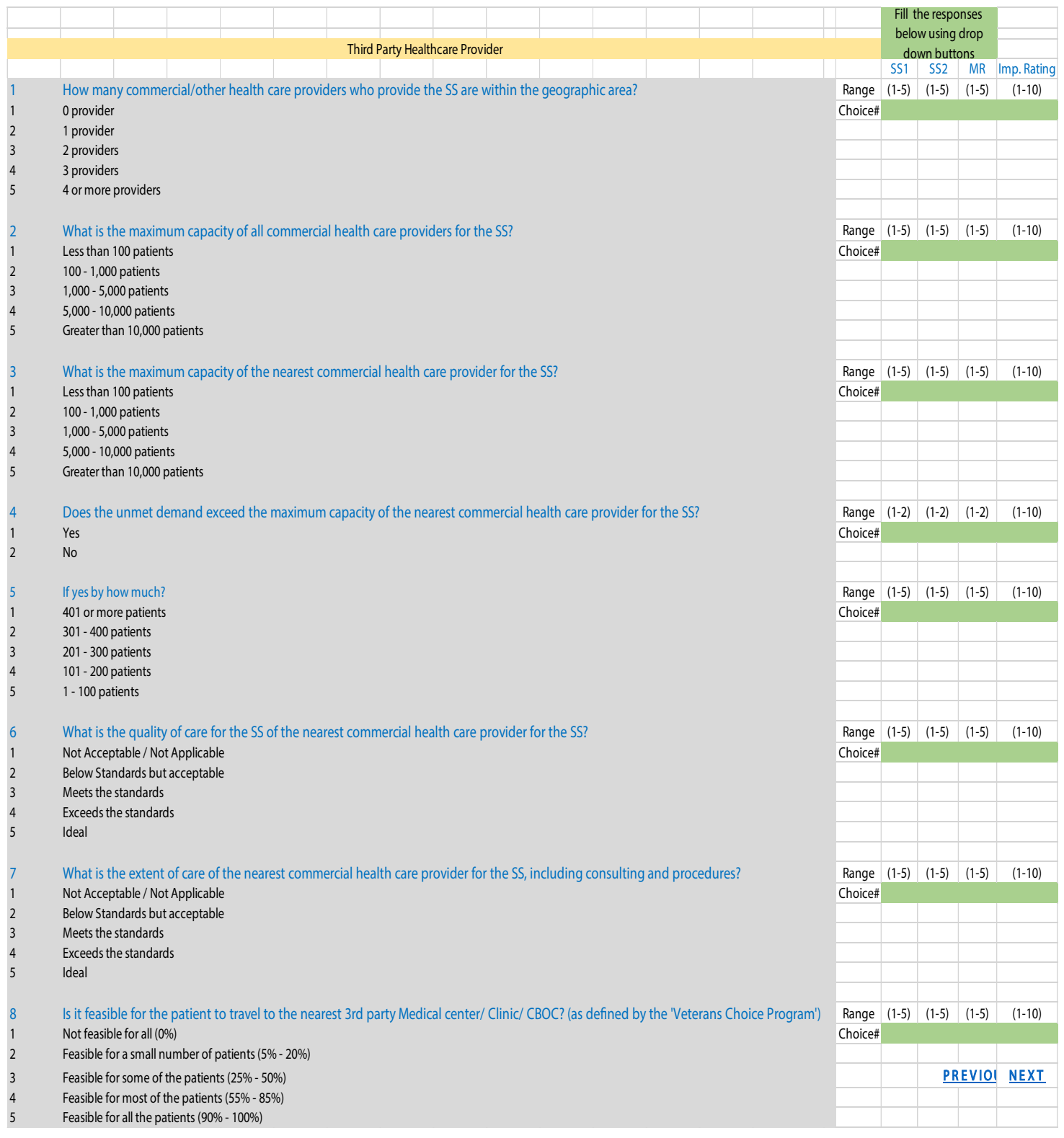

Figure 1: An example Question for understanding the methodology of scoring. 
all the remaining comparison will be based merely on comparing the quality of the winning solutions that were determined using the scoring procedure. The comparison would be made based on cost to VA, cost to veterans; distance travelled by patients and long term sustainability. The comparison of the quality of the winning solutions is outside the scope of the tool as well as this study.

\section{Typical Questionnaire}

This is a Decision Making Tool (DMT) to assist engineering managers at VA to compare between specialty services for purpose of distribution. This document is divided into several sheets. The first section is for pre-screening, then the next three sections are for the categories representing the three possible solutions we are considering for distributing the specialty service specified in three categories; $\mathrm{C} 1$ ) using an existing VA facility; C2) using a third party facility; and C3) constructing a new facility. The last section is for results, where a user can see the scores and the decisions related to each category with respect to each specialty service. There are some hidden sheets for the used for the evaluation and calculation purpose. The questions in each category are designed to determine the incentives that are present for using the category's solution with a given specialty service. The questions in each category are designed to determine the incentives that are present for using the category's solution with a given specialty service.

Question Score $=($ Answer for SS - Answer that is minimally required $)^{\star}$ Question Rating

\section{Category Score $=$ Sum of Question Scores within each category}

To calculate the score we used Excel Visual Basic Programming. Directions for the filling the Questionnaire in excel are provided further below in the paper. To look to an example for the question format refer to (Figure 2).

\section{Initial Screening}

The initial screening has one purpose; evaluating the basic need for distributing the SS. For initial screening, a specialty service is allowed to be further investigated if there is a demand in the area but some or all of it is unmet, or if the quality of service does not meet the standards. The specialty Service will not be allowed to be further investigated if there is no demand for it in the area or if all its demand is already being met with high quality service. A positive score indicates incentive to allow the specialty service to be further investigated, a negative score indicates incentive to not allow the specialty service to be further investigated. To refer to the questions in this category please refer to (Figure 3).

\section{Facility / Availability / Infrastructure Capacity}

The High positive score in this category means that there is no VA facility which provides the SS in the geographic area or the VA facility has no capacity to absorb the unmet demand. On the other hand there are other VA medical providers, who have the capacity to absorb the unmet demand. Other important thing to be kept in mind for scoring is that these medical providers should be at the minimum average distance of at least 40 miles (Veterans Choice program) and it is feasible for the patients to travel to the providers as per the Veterans Choice program.

The High negative score means that there are VA facilities in that area which provides the SS or there is no considerable demand. Another possibility for the negative score is that there are no other VA medical providers in that area who deal with that SS or if there are medical providers, but the quality of care is not up to the mark. In all the above cases the SS cannot be distributed to the existing facility. To refer to the questions in this category please refer to (Figure 4).

\section{Third Party Healthcare Provider}

The High positive score in this category means that there is third party healthcare provider which provides the SS in that area and at the same time, they have capacity to absorb the unmet demand. On the other hand the VA medical providers, do not have the capacity to absorb the unmet demand. Other important thing to be kept in mind for scoring is that these third party healthcare should be at the minimum average distance and it is feasible for the patients to travel to them as per 'Veterans Choice program'.

The High negative score means that there are VA facilities in that area which provide the SS or there is no considerable demand. Another possibility for the negative score is that there are no third party healthcare providers in that area who deals in that SS or if there are third party healthcare, but the quality of care is not up to the mark. In all the above cases the SS cannot be distributed to the existing third party. To refer to the questions in this category please refer to (Figure 5).

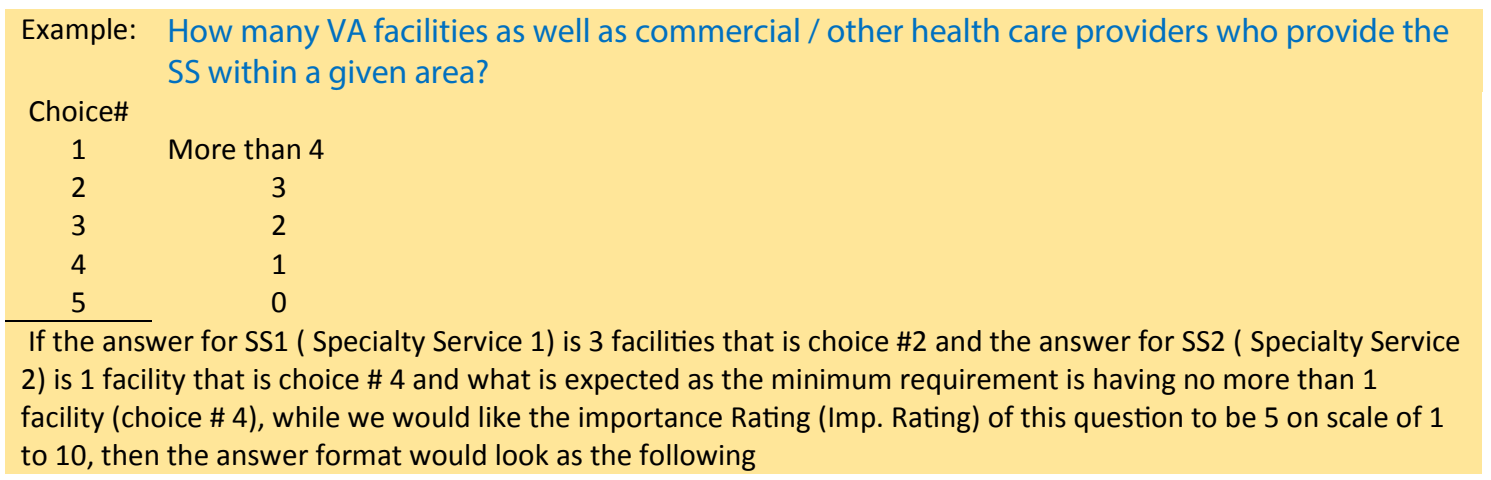

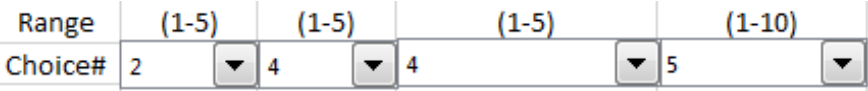

Figure 2: An example of the question format and the filling instructions 
Citation: Farahmand K, Wadhwa SS, Mostafa M, Khiabani VH (2016) Selecting Environments for Distribution of Services. Health Care: Current Reviews 4: 159. doi: 10.4172/2375-4273.1000159

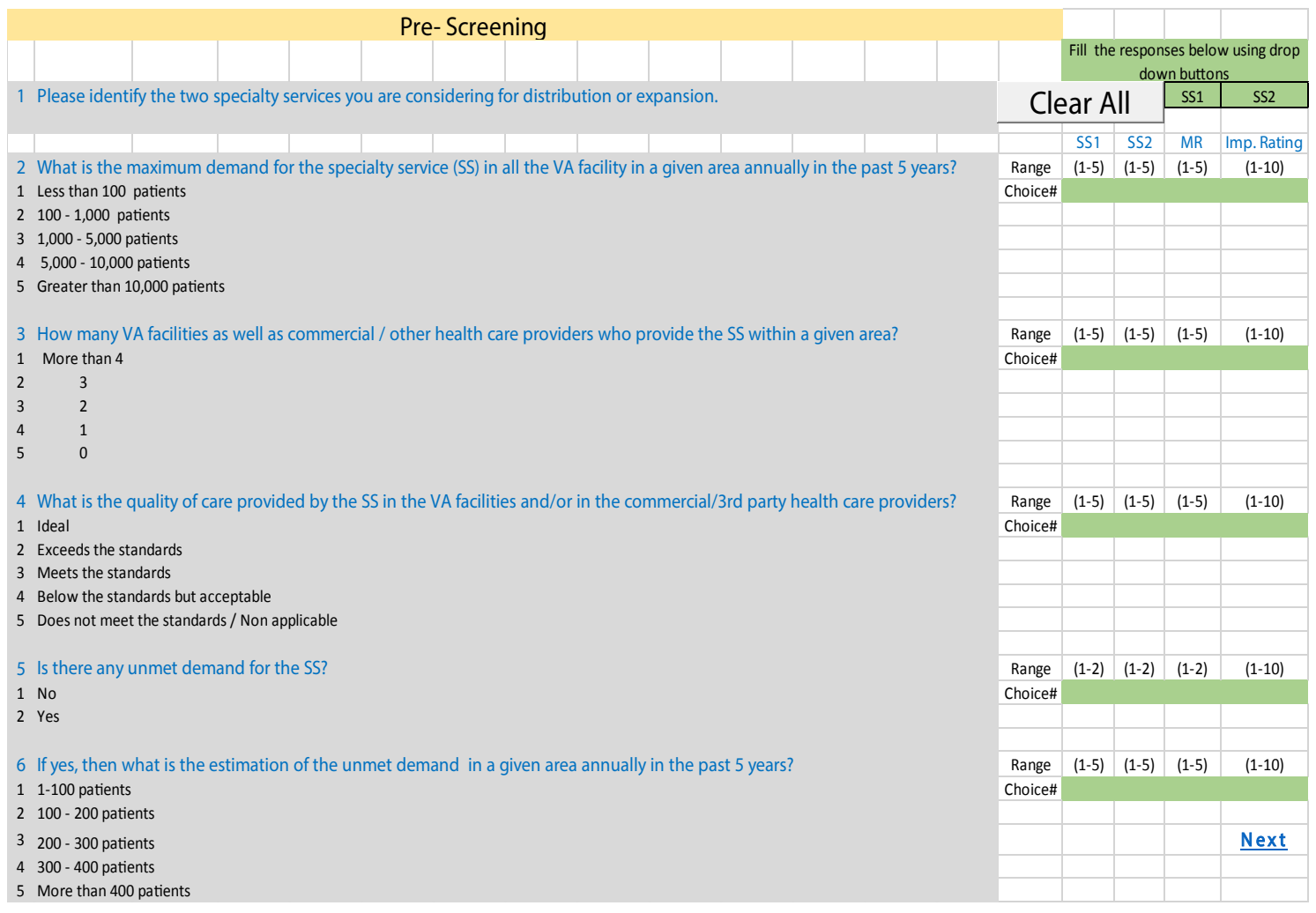

Figure 3: Questionnaire for the initial screening category.

\section{Feasibility of a New Facility}

Constructing a new facility would be considered only if the other two solutions are not applicable. Hence, the feasibility for a new facility will automatically be rejected, once one of the other two solutions is deemed viable. To refer to the questions in this category please refer to (Figure 6).

\section{Using Excel}

The decision making tool utilizes a widely available software; Microsoft Excel Workbook. Excel acts as the user interface, providing the questions and allowing the user to input the answers by choosing the answer number from a drop down list. Each category of questions is available on a separate Excel Sheet within the Excel Workbook and they are all linked through "Previous" and "Next" buttons at the end of each list of questions. The last list of questions is ended by a "Click Here to View Results" button. All user input is linked to a Response Sheet which collects all the answers from the various heets. The Response Sheet is hidden from the user and, other than the response boxes, all sheet cells are protected by a password to prevent unauthorized editing.

\section{Instructions to Fill in the Responses}

There are four response sheets pre-screening, category1, category2 and category3. The terminology used:

SS1 - Specialty Service 1

SS2 - Specialty Service 2

Min. Requirement - Minimum Requirement

Imp. Rating- Importance Rating.
1. The user should start by filling in the names of two or less specialty services on the screening category sheet.

2. The user should fill in the responses by selecting from the drop down menus within each cell in the right section of each sheet.

3. The responses will be in the form of Choice numbers.

4. The Minimum requirement is the required minimum threshold of the question for either SS1 or SS2 to measure the answer's scale of 'meeting the minimum expectations".

5. The Importance rating is the importance of the question with respect to that category.

6. At the end of the each series of questions, the user should click "NEXT" if he wishes to proceed to the next series of questions or click "PREVIOUS" if he wishes to change an answer the previous series of questions.

7. The user should click "Click Here to View Results" at the end of the last series of questions.

\section{Discussion}

\section{Example for using the Decision Making Tool}

We ran a scenario where we are looking at distributing two SS. For the sake of determining the quality of the solution given by the tool, certain assumptions are made regarding the following;

\section{Demand}

Orthopedic has low demand but the trend shows that the demand is on the rise. Podiatry, having a high demand, is already established 
Citation: Farahmand K, Wadhwa SS, Mostafa M, Khiabani VH (2016) Selecting Environments for Distribution of Services. Health Care: Current Reviews 4: 159. doi: 10.4172/2375-4273.1000159

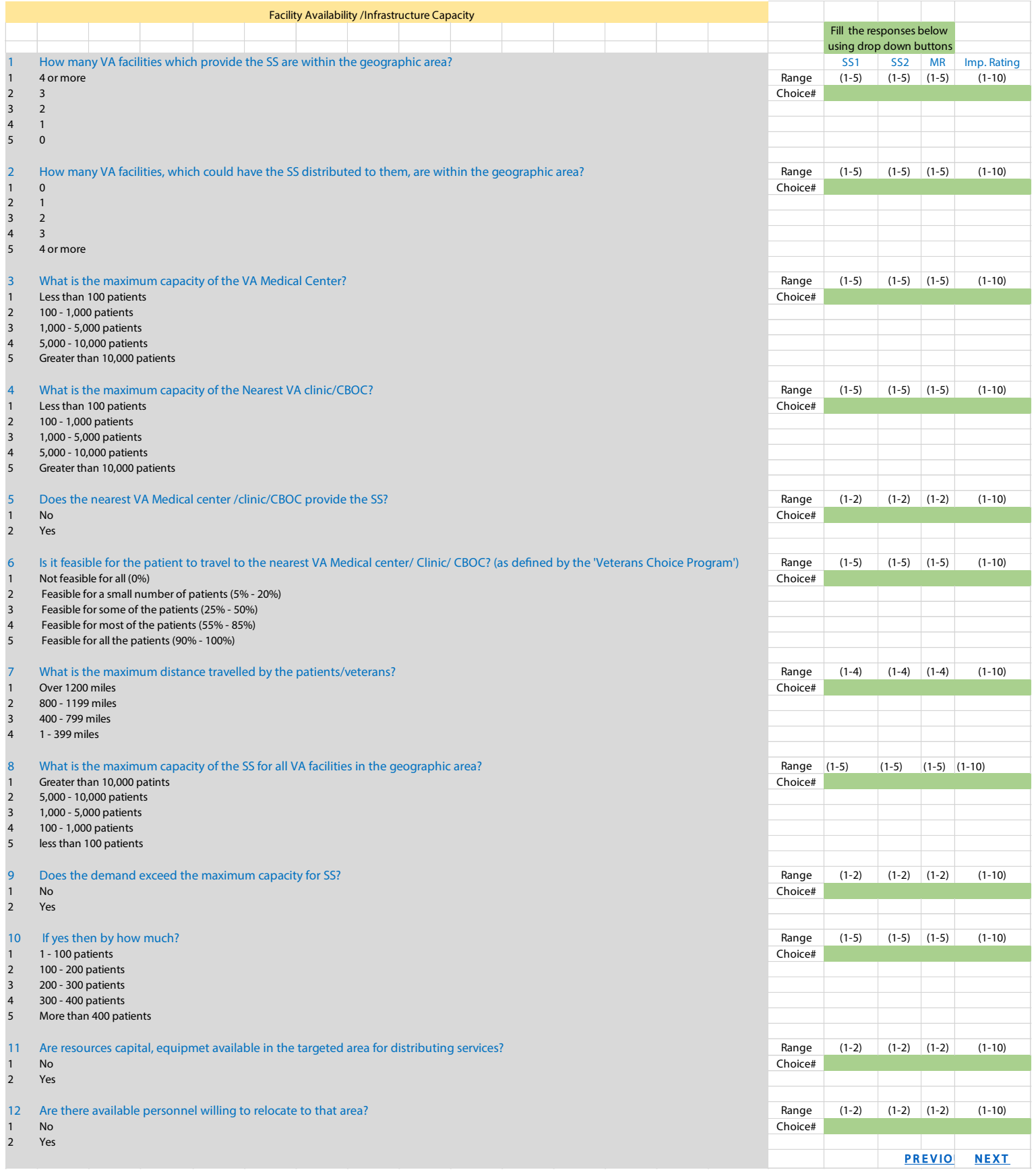

Figure 4: Questionnaire for facility availability /infrastructure capacity category.

in the area and is widely used by the patients and the demand is met. Management put the highest emphasis on meeting the demand that is higher than a pre-set threshold. The Orthopedic demand is exactly at that threshold. The demand in this case is equal to the unmet demand for the orthopedic services.

\section{Existing Medical Facilities}

\section{Service type}

Medical facilities in the area do not provide orthopedic services but they do provide podiatry services.

\section{Capacity}

There are 2 Medical facilities in the area; A VA Medical Center and a VA outpatient clinic. Generally speaking, these VA facilities maintain high capacity and there is available space for expansion. Most of the Patients have no problem traveling to the VA facilities in the area.

\section{Proximity}

The majority of the patient population are in closer proximity to the VA medical center than the outpatient clinic. 
Citation: Farahmand K, Wadhwa SS, Mostafa M, Khiabani VH (2016) Selecting Environments for Distribution of Services. Health Care: Current Reviews 4: 159. doi: 10.4172/2375-4273.1000159

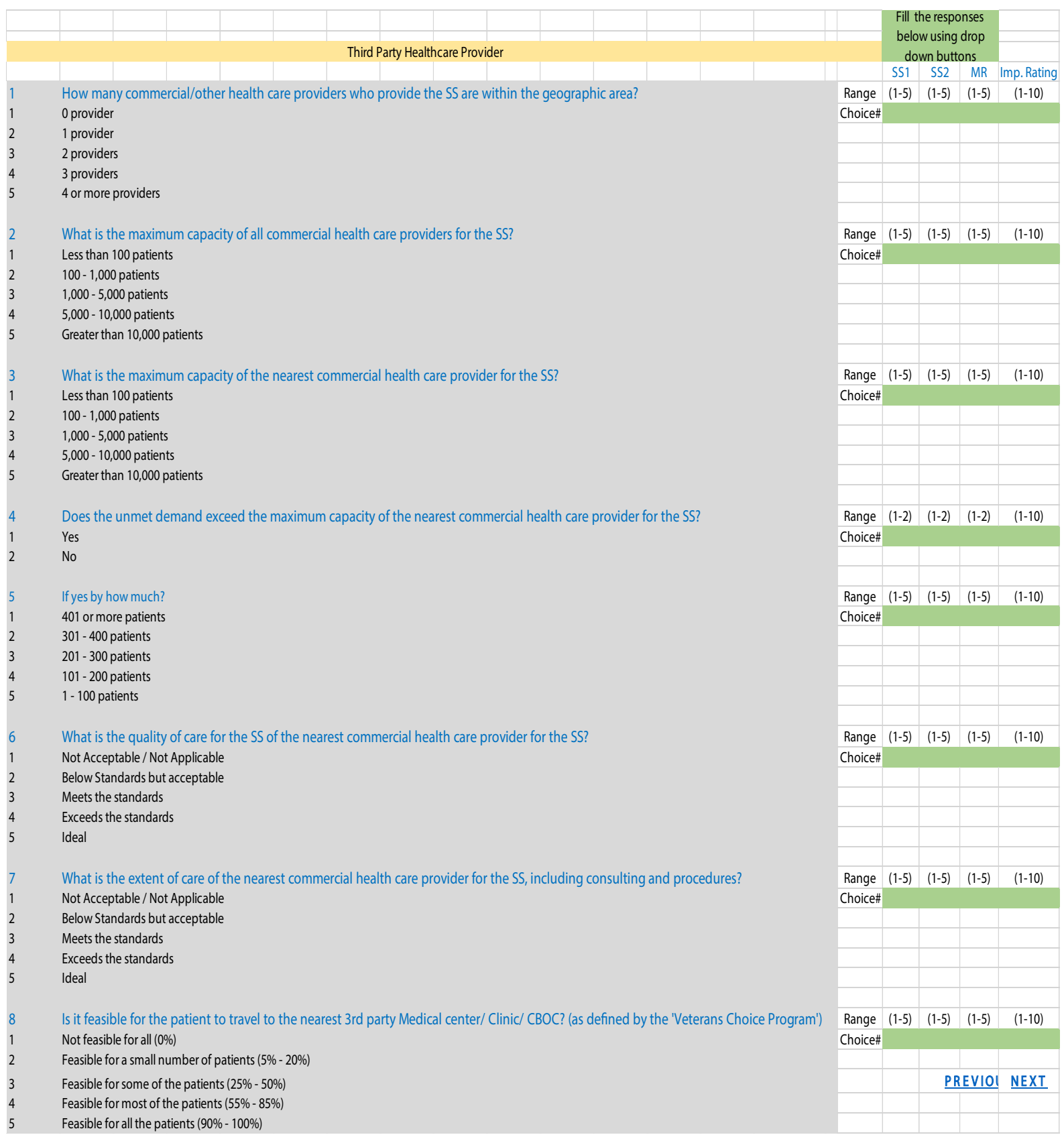

Figure 5: Questionnaire for third part healthcare provider category.

\section{Priorities for distributing to an existing VA facility}

Management put a high emphasis on the quality of care provided to the patients. Management is interested in looking into the VA facilities that provide opportunities for expansion; whether it is an expansion for the capabilities of the existing specialty service or adding a new one. An important assumption is that expansion for the capabilities of the existing specialty service would be more preferable to adding a new one as it requires a less drastic change. Meeting the standards of the Veteran's Choice Program is also on the top priority list for management.

\section{Third Party Facilities}

\section{Capacity and quality}

Third party facilities that provide podiatry in the area are two small facilities with limited capacity but exceedingly high quality care. There is one third Party provider that has been providing an exceedingly high quality orthopedic services in the area with very limited capacity.

\section{Proximity}

They are a little further from the patients than the VA facilities. 
Citation: Farahmand K, Wadhwa SS, Mostafa M, Khiabani VH (2016) Selecting Environments for Distribution of Services. Health Care: Current Reviews 4: 159. doi: 10.4172/2375-4273.1000159

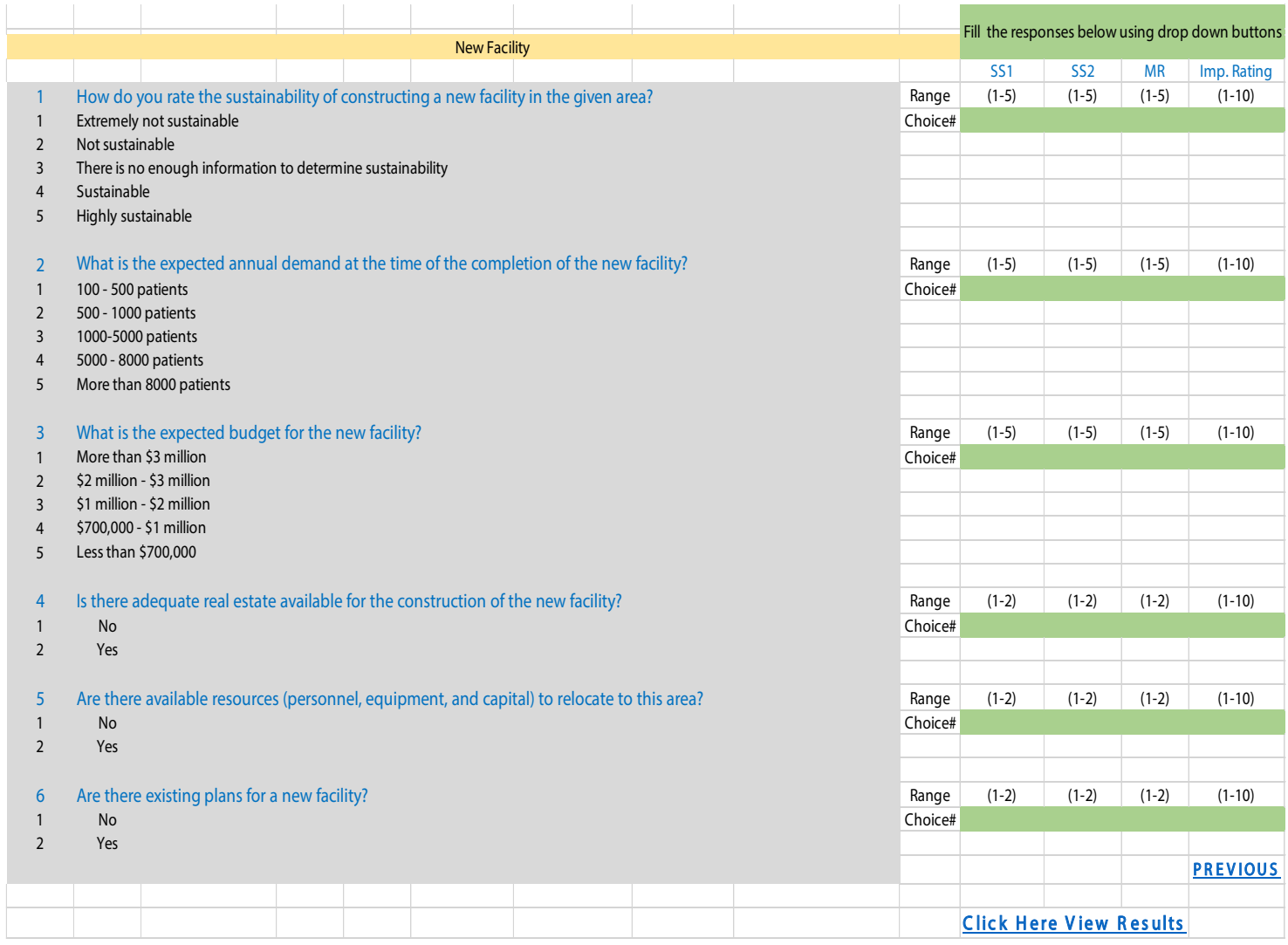

Figure 6: Questionnaire for the new facility category.

\section{Priorities for distributing to a third party provider}

Management is more interested in using third Party facilities when there is more than one third Party provider in order to leverage the negotiations with the third Party providers.

\section{New Facility}

\section{Sustainability}

Given the current trend of increase in demand for orthopedic services, constructing a new facility for orthopedic is sustainable. A new facility is considered sustainable for podiatry as well based on the assumption that there would be a huge surge in demand (due to an end to a war or a massive recall of soldiers from overseas) by the time of the facility's completion.

\section{Resources and planning}

There are viable plans for constructing a new facility. Real estate is available for construction, as well as capital and personnel that are willing / feasible for relocation.

\section{Top Priorities}

The management places heavy emphasis on fulfilling any unmet demand, minimizing waste, insuring that the requirements of the Veteran's Choice Program are met and maintaining a certain level of quality care for the patients.

\section{Results and Discussion}

Based on the above mentioned assumptions, the answers were inputted into Excel and the results were obtained and provided below. The results include:

1. Overall Score for each solution regarding each specialty service

\section{A final decision for each specialty service}

Orthopedic department scored the highest for the decision to "distribute to an existing medical facility", which goes along with what we would conclude by intuition. Distributing to a third party provider was shown to be a viable option due to its extreme high quality care and the partial availability of capacity but given its limited capacity that would not allow the fulfillment of all the unmet demand, regardless of its quality care, distributing to the high capacity medical facilities proved to be a better option. The results for the orthopedic solutions are listed in Table1.

Podiatry scored well in all the categories but the final decision was that it is not viable for distribution. The reason for this decision is that it did not pass screening since currently all of the podiatry demand is fulfilled. However, using third party providers is shown to be an extremely viable option if management decides to divert some

\begin{tabular}{|l|l|l|l|l|}
\hline Category & Pros & Avg Imp. & Cons & Avg. Imp. \\
\hline VA Facility & High Capacity & 10 & & \\
\hline & Proximity & 10 & & \\
\hline & $\begin{array}{l}\text { Availability of } \\
\text { resources }\end{array}$ & 8 & & 10 \\
\hline $\mathbf{3}^{\text {rd }}$ party & High Quality & 10 & Extremely low capacity & 10 \\
\hline & Sustainable & 10 & Other options are more viable & N/A \\
\hline
\end{tabular}

Table 1: Orthopedic solutions pros and cons. 


\begin{tabular}{|l|l|c|l|c|}
\hline Category & Pros & $\begin{array}{c}\text { Avg } \\
\text { Imp. }\end{array}$ & Cons & $\begin{array}{c}\text { Avg. } \\
\text { Imp. }\end{array}$ \\
\hline VA Facility & High Capacity & 10 & No Unmet Demand & 10 \\
\hline & Proximity & 10 & & \\
\hline & Availability of resources & 8 & & 10 \\
\hline $\mathbf{3}^{\text {rd }}$ party & High Quality & 10 & Low Capacity & 10 \\
\hline New Facility & $\begin{array}{l}\text { Highly Sustainable (only if the } \\
\text { demand surges) }\end{array}$ & 10 & $\begin{array}{l}\text { The demand is } \\
\text { currently fully met }\end{array}$ & 10 \\
\hline & $\begin{array}{l}\text { Availability of resources and } \\
\text { planning }\end{array}$ & 8 & & \\
\hline
\end{tabular}

Table 2: Podiatry solutions pros and cons.

of the demand in order to free some resources. A new facility could also be considered a viable option if a sudden surge in demand could be foreseen in the far future. The results for the orthopedic solutions are listed in Table 2. Avg Imp. (Importance) is the importance rating on an average given by the user (manager) for the deciding factors of a category based on its importance to the particular specialty service.

\section{Conclusion}

We are still far from a definitive tool, if such a tool is indeed possible, but believe that the tool makes a contribution in two major ways. The first $\dot{\mathbf{s}} \mathbf{b}$ fill a es earch pa ctice gp in evi dence-based health care management by providing a practical approach for the method comparison and selection. Not only can the tool help health care professionals commission more appropriate modelling work, but may also assist health care modelling consultants and researchers to expand their modelling repertoire in order to meet the diverse needs of healthcare clients. The second contribution is that the development of the tool has also highlighted significant gaps in the existing literature, which could be usefully filled. There are four solutions for distributing specialty service; 1) distributing to an existing facility; 2) using a third party provider; 3) building a new facility; or 4) a combination of some of them. Using this tool we were able to choose the best solution for the two specialty services orthopedics and podiatry. This tool uses MS office excel, where a healthcare manager has to input all the responses and on the click of a button he can get the scores for all the solutions. The solution with the highest score for each specialty service wins.

In spite of many debates and discussions throughout the tool development process, we identified the following two research areas to be further addressed. First, this tool is based on the previous data for the demand of a specialty service in a geographic area. But in future, if the demand surges due to any this tool may be inefficient. So, future work requires to incorporate some forecasting method that can measure the demand and so to make this tool more resilient. Second, the final solutions for the distribution of services have to further analyzed and compared on the parameters of cost to the facility, cost to the patient and sustainability in order to confirm their practical implementation. In summary, we believe the tool adds value to the scarce existing literature on the selection of environments for the distribution of services.

\section{References}

1. Anthony David Bristol (2005) Everyone is; should healthcare? Outsourcing issues in strategic Planning, organizational culture, and organizational ethics. A Dissertation submitted to the University of Texas Health Science Center at Houston School of Public Health.

2. Rana Zadeh, Hessam Sadatsafavi, Ryan Xue (2015) Evidence-based and value-based decision making about healthcare design: an economic evaluation of the safety and quality outcomes. Health Environments Research and Design Journal 8: 58-76.

3. Yiye Zhu, Jianping Shen, Yinsheng Li (2014) A Human-Centric User Mode for Intelligent Healthcare. IEEE 11th International Conference on e-Business Engineering.

4. Beer, Martin, Keefe, Terry, Snelling (2008) Using trading games to interpret a health environment $2-5$

5. Gyuchan Thomas Jun, James Ward, Zoe Morris, John Clarkson (2009) Health care process modelling: which method when? International Journal for Quality in Health Care 3: 214-224.

6. Gyuchan T Jun, Zoe Morris, Tillal Eldabi, Paul Harper, Aisha Naseer et al. (2011) Development of modelling method selection tool for health services management: From problem structuring methods to modelling and simulation methods.BMC Health Services Research 11: 108. 